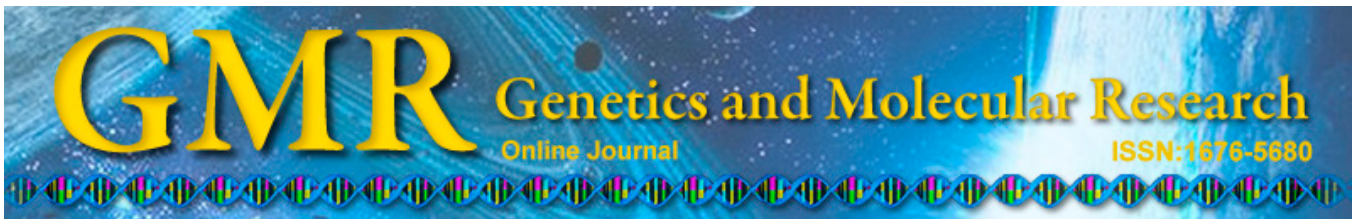

\title{
Exploring laccase genes from plant pathogen genomes: a bioinformatic approach
}

\author{
B.Z. Feng ${ }^{1}$, P.Q. Li $^{1}$, L. Fu ${ }^{2}$ and X.M. Yu ${ }^{2}$ \\ ${ }^{1}$ Department of Life Sciences, Yuncheng University, Yuncheng, China \\ ${ }^{2}$ Shandong Institute of Pomology, Taian, China \\ Corresponding author: B.Z. Feng \\ E-mail: fengbaozhen@126.com
}

Genet. Mol. Res. 14 (4): 14019-14036 (2015)

Received April 10, 2015

Accepted May 25, 2015

Published October 29, 2015

DOI http://dx.doi.org/10.4238/2015.October.29.21

\begin{abstract}
To date, research on laccases has mostly been focused on plant and fungal laccases and their current use in biotechnological applications. In contrast, little is known about laccases from plant pathogens, although recent rapid progress in whole genome sequencing of an increasing number of organisms has facilitated their identification and ascertainment of their origins. In this study, a comparative analysis was performed to elucidate the distribution of laccases among bacteria, fungi, and oomycetes, and, through comparison of their amino acids, to determine the relationships between them. We retrieved the laccase genes for the 20 publicly available plant pathogen genomes. From these, 125 laccase genes were identified in total, including seven in bacterial genomes, 101 in fungal genomes, and 17 in oomycete genomes. Most of the predicted protein models of these genes shared typical fungal laccase characteristics, possessing four conserved domains with one cysteine and ten histidine residues at these domains. Phylogenetic analysis illustrated that laccases from bacteria and oomycetes were grouped into two distinct clades, whereas fungal laccases clustered in three main clades. These results
\end{abstract}


provide the theoretical groundwork regarding the role of laccases in plant pathogens and might be used to guide future research into these enzymes.

Key words: Laccase; Plant pathogen; Bioinformatics; Bacteria; Fungi; Oomycetes

\section{INTRODUCTION}

Laccases (EC No. 1.10.3.2) are widely distributed oxidoreductases that catalyze the biological oxidation-reduction of polyphenols with a concomitant reduction of molecular oxygen to water. They belong to the multi-copper oxidases (MCOs), which are characterized by having four bound copper atoms (McGuirl and Dooley, 1999). These copper atoms are classified as T1 (blue copper), T2, or T3 according to their spectroscopic characteristics (Quintanar et al., 2007). Cu binding domains are highly conserved among laccases. Numerous genes coding for laccase proteins have been cloned and characterized from various sources (Zhao and Kwan, 1999; Litvintseva and Henson, 2002; Hoegger et al., 2004; Baldrian, 2006; Kilaru et al., 2006; Courty et al., 2009; Lettera et al., 2010; Levasseur et al., 2010; Feng and Li, 2012, 2013, 2014). Laccases have received much attention due to their broad substrate specificity, making them useful in wood processing and the textile industry (Rodriguez Couto and Toca Herrera, 2006).

Laccases are found in plants as well as in various microorganisms. Reports have shown that laccases exist as a gene family in bacteria (Ausec et al., 2011), fungi (Hoegger et al., 2004; Courty et al., 2009; Cázares-Garcia et al., 2013) and oomycetes (Feng and Li, 2012). Plant pathogenic fungi also produce many kinds of laccases. In Rhizoctonia solani, a soil-born fungus infecting a wide range of crop plants, four laccase genes have been identified (Wahleithner et al., 1996). In Gaeumannomyces graminis var. tritici, an important root pathogen of cereals that causes take-all disease, three laccase genes have been identified (Litvintseva and Henson, 2002). In Botrytis cinerea, a broad-host-range necrotrophic pathogen, two laccase genes have been cloned and characterized (Schouten et al., 2002). In Fusarium proliferatum, an opportunistic pathogen isolated from wheat, three laccase genes have been isolated (Kwon and Anderson, 2001). Cañero and Roncero (2008) isolated and characterized six laccase genes, $1 \mathrm{cc} 1,1 \mathrm{cc} 2, \operatorname{lcc} 3, \operatorname{lcc} 4,1 \mathrm{cc} 5$, and $1 \mathrm{cc} 9$, from the vascular wilt fungus Fusarium oxysporum. In the chestnut blight fungus Cryphonectria parasitica, the laccase gene lac3 (GenBank accession No. AY994151) consisting of 567 amino acids was isolated (Chung et al., 2008). In oomycetes, which share morphological features with some fungal plant pathogens but fall within the kingdom Stramenopila (Yoon et al., 2002), a number of laccase-like genes have been identified in the genus Phytophthora. Four laccaselike genes were identified in the $P$. capsici genome, whereas six were identified in the $P$. sojae genome, and eight in the $P$. ramorum genome (Feng and Li, 2012). These three species are the cause of blight and crown rot as well as of stem, leaf, and fruit lesions on many plants (Erwin and Ribeiro, 1996). However, very few reports have been published to date that document pathogenic bacterium laccase genes.

Studies have documented that laccases display diversity in biological function including lignin degradation and fungal morphogenesis, and in industrial applications (Litvintseva and 
Henson, 2002; Baldrian, 2006). However, in the last decade some evidence has suggested that this enzyme plays different roles in fungal pathogenesis. In the animal pathogen Cryptococcus neoformans, laccases are involved in melanin synthesis (Zhu and Williamson, 2004) and are thus considered as important virulence factors (Zhu et al., 2001). Significant reductions in laccase activities have been associated with hypovirulence in virus-infected strains of the chestnut blight fungus C. parasitica (Rigling and Van Alfen, 1991, 1993; Chung et al., 2008), whereas the phytopathogenic fungus $F$. oxysporum possesses two intracellular laccases, Lcc1 and Lcc3, which might be involved in the protection of the fungus against oxidative stress and toxic compounds (Cañero and Roncero, 2008). However, although laccase isoenzymes are encoded by gene families in many pathogenic fungi and oomycete species, to our knowledge little information about their function has been elucidated.

As more genomes are sequenced and the genes annotated therein, it has become suitable to perform bioinformatic analysis among different species. Genome-wide comparisons among these pathogens will enable the comparative analyses of functional genes and will reveal insights into the processes of pathogenesis and biotrophy.

This study was the first to evaluate plant pathogen laccases at the level of 1) the distribution of laccase genes within bacteria, fungi, and oomycetes; 2) the diversity of the genes for these species; 3) the structural characteristics of their coded proteins; and 4) the phylogenetic relationships of the putative laccases. This approach provided the theoretical ground for new hypotheses about the roles of laccases in plant pathogens and might guide the future research of these interesting and biotechnologically important enzymes.

\section{MATERIAL AND METHODS}

\section{Data sets}

We downloaded the 20 pathogen genomes reported available from known websites [the Broad Institute and the Department of Energy (DOE) Joint Genome Institute]. Five bacterial genomes were obtained from the Broad Institute (http://www.broadinstitute.org/) including Erwinia amylovora CFBP1430, Xanthomonas oryzae pv. oryzae KACC 10331, X. oryzae pv. oryzicola BLS256, Pseudomonas syringae DC3000, and P. syringae pv. syringae B728a. We also obtained 11 fungal genomes from the Broad Institute as well: $C$. parasitica, Fusarium graminearum PH-1, F. oxysporum f. sp lycopersici 4287, F. verticillioides 7600-3, Gibberella zeae PH-1, Magnaporthe oryzae 70-15, Puccinia graminis var. tritici, Ustilago maydis, Rhizopus oryzae, Sclerotinia sclerotiorum, and Verticillium dahliae VdLs.17. Databases of pathogenic oomycete genomes included four available species from the genus Phytophthora ( $P$. sojae genome sequence assembly database V3.0, $P$. capsici genome sequence assembly database V1.1, P. ramorum genome sequence assembly database V1.1, and P. parasitica) that were downloaded from the DOE Joint Genome Institute (http:// genome.jgi.doe.gov/).

\section{Genome analysis}

From the National Center for Biotechnology Information (NCBI) GenBank database, 
we obtained the sequences of various multi-copper oxidases, including those of Cucurbita maxima (GenBank accession No. BAA02123), Melanocarpus albomyces (GenBank accession No. CAE00180), Myrothecium verrucaria (GenBank accession No. BAA09528), and Saccharomyces cerevisiae (GenBank accession No. AAA64929), which were used as queries to search for laccase genes in the 20 pathogen genomes. Various members of the MCO family were used to assure the identification of all possible laccases in the genomes analyzed based on the identity of copper binding sites. In addition, only the gene and amino acid sequences from crystallized proteins, for which there is no doubt regarding their identity, were used in this analysis. Sequences were selected for the presence of the four preserved copper binding motifs characteristic of all MCOs.

\section{Sequence analysis}

The protein sequences of predicted laccase genes were submitted to SignalP v3.0 (http://www.cbs.dtu.dk/services/SignalP/) (Bendtsen et al., 2004) for secreted signal peptide prediction, whereas NetNGlyc 1.0 (http://www.cbs.dtu.dk/services/NetNGlyc/) was used to determine the sites of N-glycosylation (Asn-XXX-Ser/Thr). Protein domain and motif analysis was conducted using the NCBI conserved domain database (http://www.ncbi.nlm. nih.gov/Structure/cdd/cdd.shtml) and the SMART software (http://smart.embl-heidelberg.de/) (Marchler-Bauer and Bryant, 2004; Marchler-Bauer et al., 2009). Multiple alignment of all retrieved sequences was performed using ClustalX (Larkin et al., 2007) to identify and remove duplicate entries.

\section{Phylogenetic analysis}

To generate phylogenetic tree, all predicted laccase genes from the different pathogens were used, as shown in Tables 1-3. Multiple alignments of these sequences were performed using Clustal X (2.0). Phylogenetic trees were generated by neighbor-joining, as implemented in PAUP* 4.0 Beta (Sinauer Associates, Sunderland, MA, USA) with the default parameters. Nodal support of the trees was estimated by bootstrapping (Felsenstein, 1985) with 1000 pseudo-replicate data sets.

\section{RESULTS}

\section{Identification of laccase genes in plant pathogen genomes}

Four laccase sequences, including those of C. maxima (GenBank accession No. BAA02123), M. albomyces (GenBank accession No. CAE00180), M. verrucaria (GenBank accession No. BAA09528), and S. cerevisiae (GenBank accession No. AAA64929) were used to search the 20 genomes using the TBLASTn program and an expected (E) value cutoff $<10^{-15}$. A total of 125 predicted gene models were retrieved under these conditions, as shown in Tables 1-3. In bacteria, seven laccase genes were searched as shown in Table 1; in fungi, 101 laccase genes occurred, which are displayed in Table 2; and in oomycetes, 17 paralogous genes were retrieved and are shown in Table 3 . 
Table 1. Summary of laccase genes in the genomes of bacterial plant pathogens.

\begin{tabular}{|c|c|c|c|c|c|}
\hline Species name & Genome position ${ }^{\mathrm{a}}$ & Designated gene name & Protein length & SignalP length ${ }^{\mathrm{b}}$ & $\begin{array}{l}\text { N-glycosylation } \\
\text { Asn-X-Ser/Thr }\end{array}$ \\
\hline \multirow[t]{2}{*}{ Erwina amylovora } & 292489271 & ealac1 & 536 & nd & nd \\
\hline & 292486950 & ealac2 & 474 & nd & nd \\
\hline Xanthomonas oryzae pv. oryzae & 58580379 & xplac1 & 638 & nd & nd \\
\hline Xanthomonas oryzae pv. oryzicola & 384420792 & xplac2 & 622 & nd & nd \\
\hline \multirow[t]{3}{*}{ Pseudomonas syringae pv. syringae } & 66044816 & pseulac1 & 592 & nd & nd \\
\hline & 66044740 & pseulac2 & 606 & nd & nd \\
\hline & 66044514 & pseulac3 & 457 & nd & nd \\
\hline
\end{tabular}

${ }^{a}$ The genome position refers to the laccase gene position in the plant pathogenic bacterium genome sequence assembly database (Erwinia amylovora CFBP1430, Xanthomonas oryzae pv. oryzae KACC 10331, Xanthomonas oryzae pv. oryzicola BLS256, and Pseudomonas syringae pv. syringae B728a); ${ }^{b}$ signal peptide lengths were

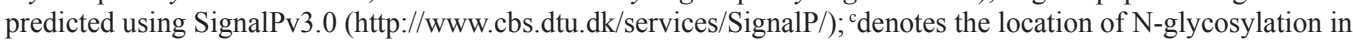
amino acid sequences from laccases; N-glycosylation sites were predicted using the NetNGlyc 1.0 Server (http:// www.cbs.dtu.dk/services/NetNGlyc); nd, not detected.

Table 1 shows the search results in the available pathogenic bacterial genomes; however, only four genomes contained laccases, as no hits were found in the P. syringae DC3000 genome database (data not shown). In E. amylovora, two laccase genes were identified and were designated ealac1 and 2; in $X$. oryzae pv. oryzae and $X$. oryzae pv. oryzicola, two laccase genes occurred and were named xplac1 and 2, respectively; and in $P$. syringae pv. syringae, three paralogous genes were retrieved and were designated pseulac1-3.

The occurrence of laccase in pathogenic fungi is shown in Table 2. In $C$. parasitica, which causes chestnut blight, 16 laccase genes were found and were named cplac1-16. Fusarium is a large genus of filamentous fungi widely distributed in soil and in association with plants. The genus includes a number of economically important plant pathogenic species, e.g., F. graminearum commonly infects barley in humid conditions. Results showed that 11 laccase members appeared in this genome, which were named fglac1-11. Additionally, in F. oxysporum $f$. sp lycopersici, a total of 14 paralogous genes were retrieved and were designated folac1-14. In F. verticillioides, a fungus that is one of the most prevalent molds on harvested maize throughout the world, 14 genes were found and named fvlac1-14. In G. zeae, which causes a devastating disease on wheat and barley, four genes were found (gzlac1-4). In M. oryzae, an important pathogen that causes blast disease or blight disease on agriculturally important cereals including rice, wheat, rye, barley, and pearl millet, a total of 13 laccases were identified. In P. graminis var. tritici, the causal agents of wheat and barley stem rust (black rust), eight genes were identified. In $U$. maydis, a basidiomycete fungal pathogen that induces tumors on maize and teosinte, six genes were found (umlac1-6). In R. oryzae, a common saprobic fungus on plants, four genes were found (rdlac1-c4). S. sclerotiorum, which is among the world's most successful and omnivorous fungal plant pathogens and exhibits a host range of greater than 400 plant species, contained seven laccase genes according to our analysis (sslac1-7). In $V$. dahliae, one of the causal agents of vascular wilt in numerous economically important plants that causes wilting of all or only parts of the host including olive and maple trees, cotton, tomatoes and potatoes, and also ornamentals, four laccase genes were retrieved (vdlac1vdlac4). 
B.Z. Feng et al.

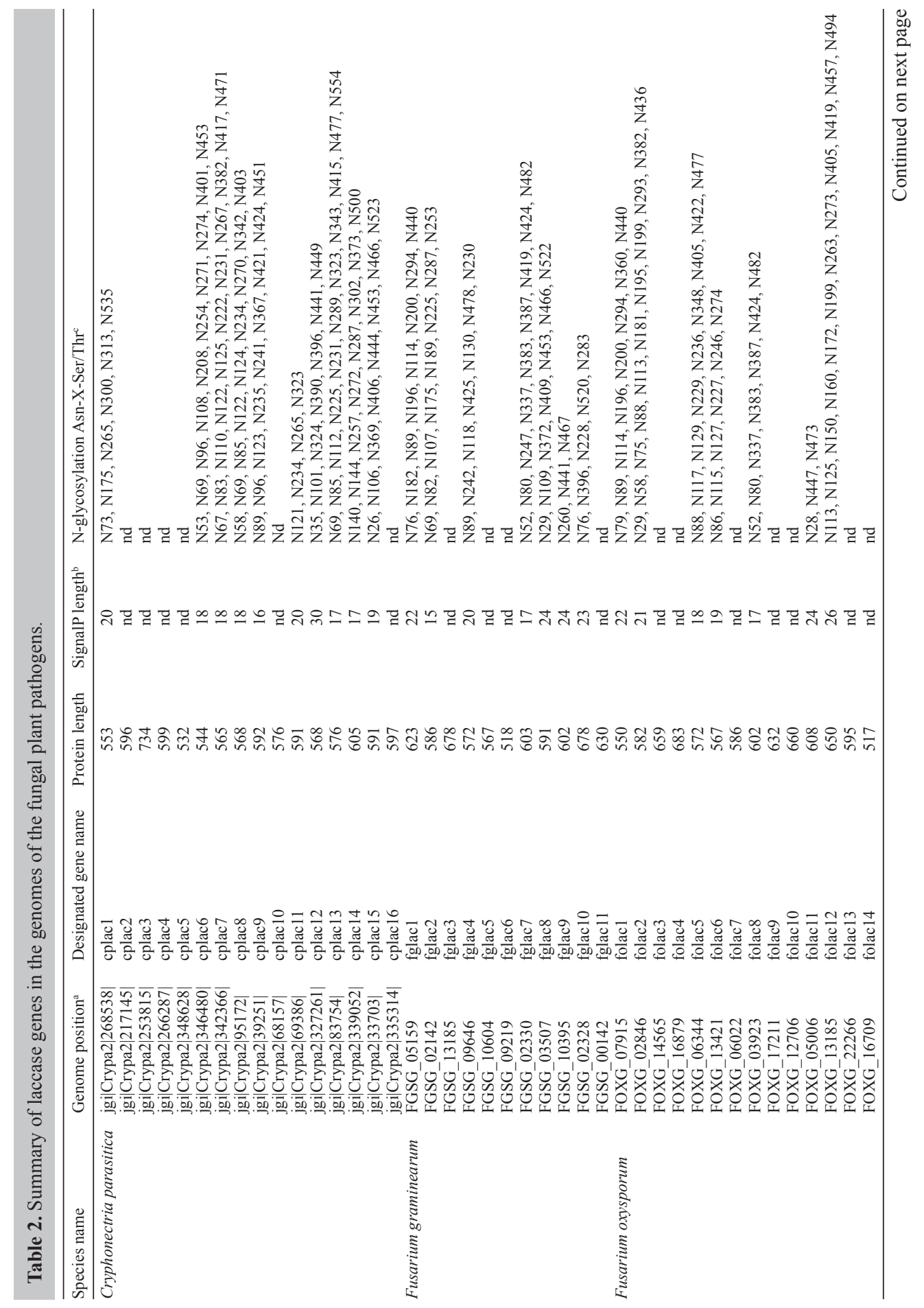


Bioinformatics of laccase genes from plant pathogen genomes

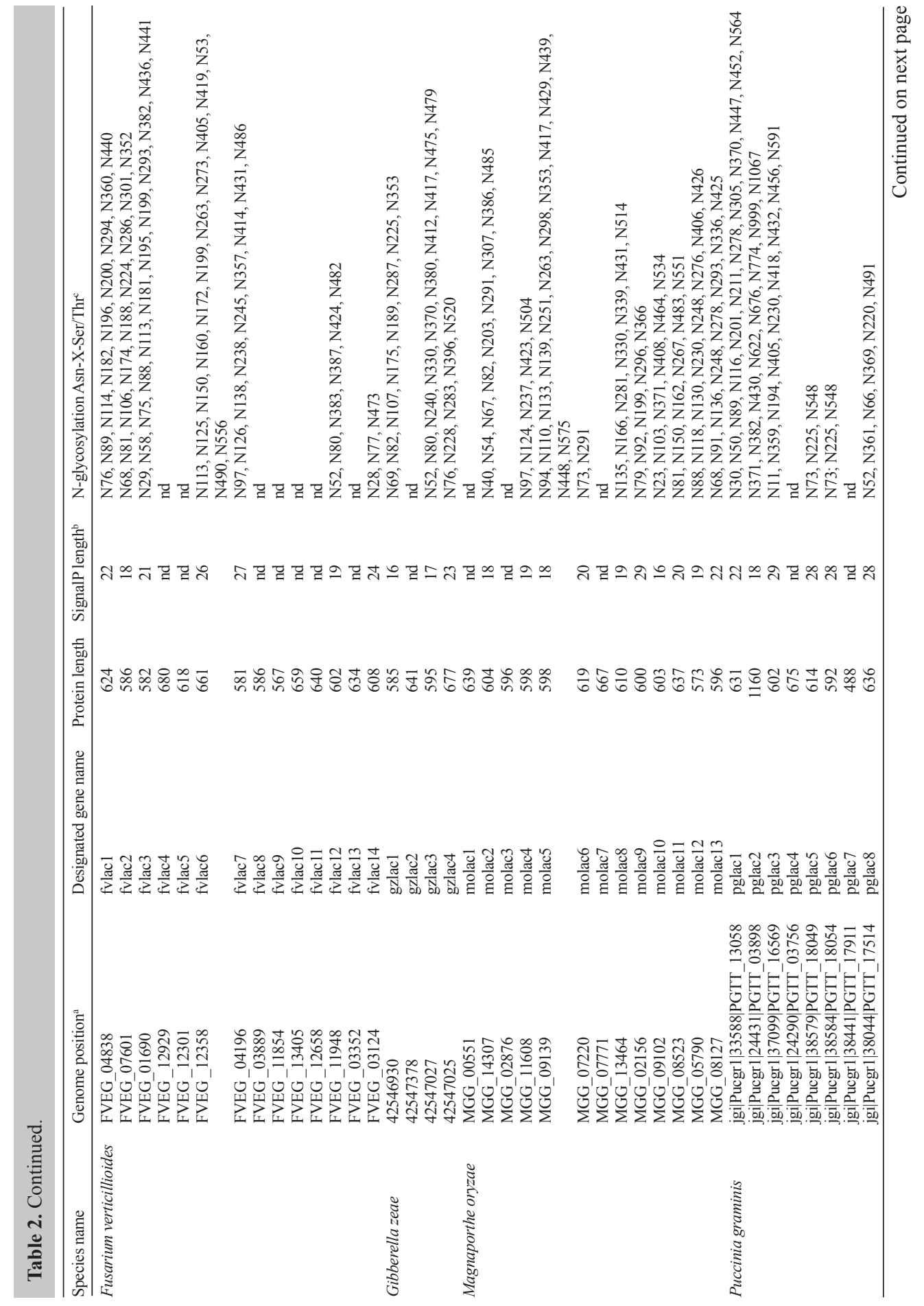

Genetics and Molecular Research 14 (4): 14019-14036 (2015)

CFUNPEC-RP www.funpecrp.com.br 
B.Z. Feng et al.

14026

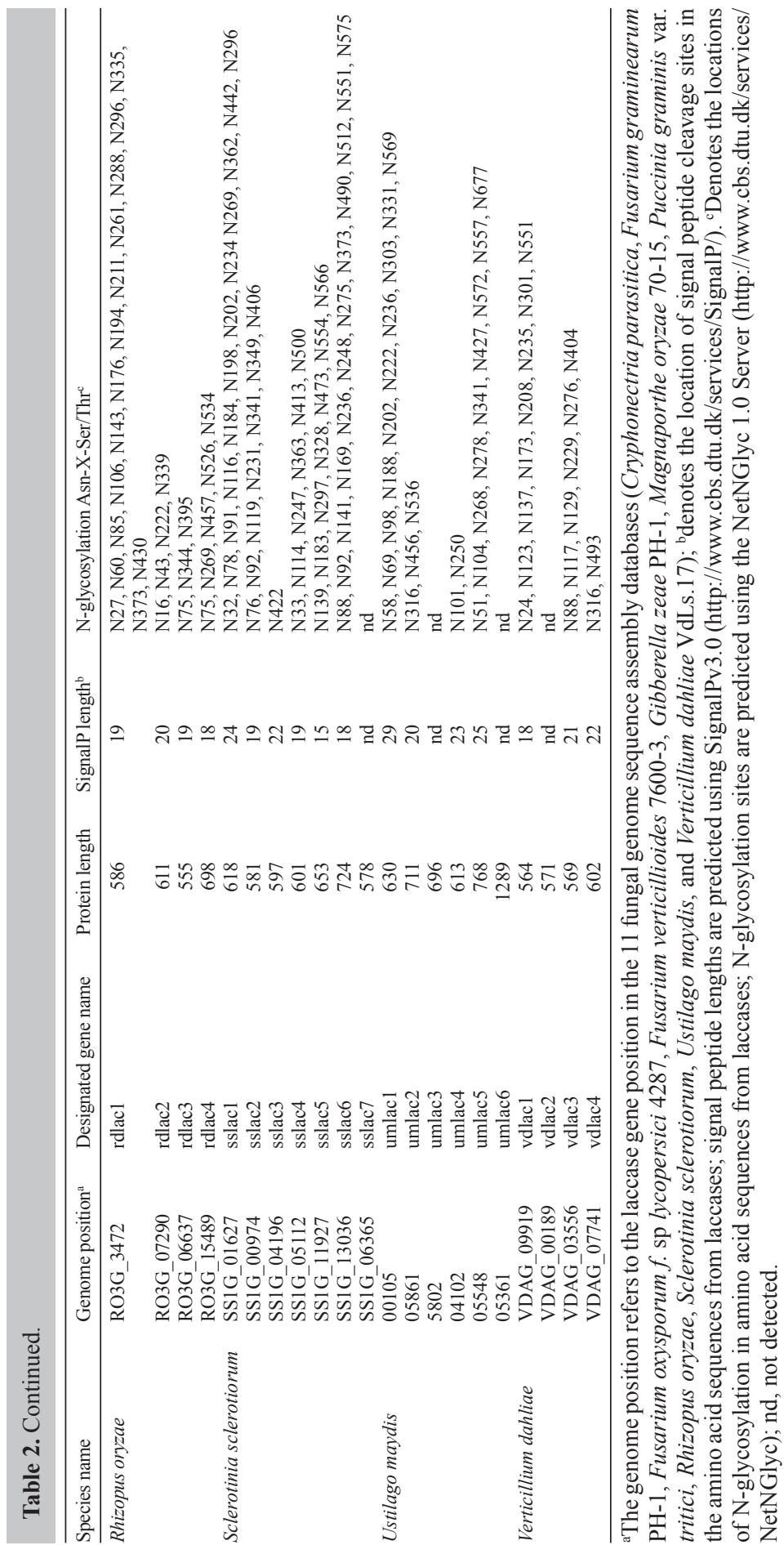

Genetics and Molecular Research 14 (4): 14019-14036 (2015)

CFUNPEC-RP www.funpecrp.com.br 
Bioinformatics of laccase genes from plant pathogen genomes

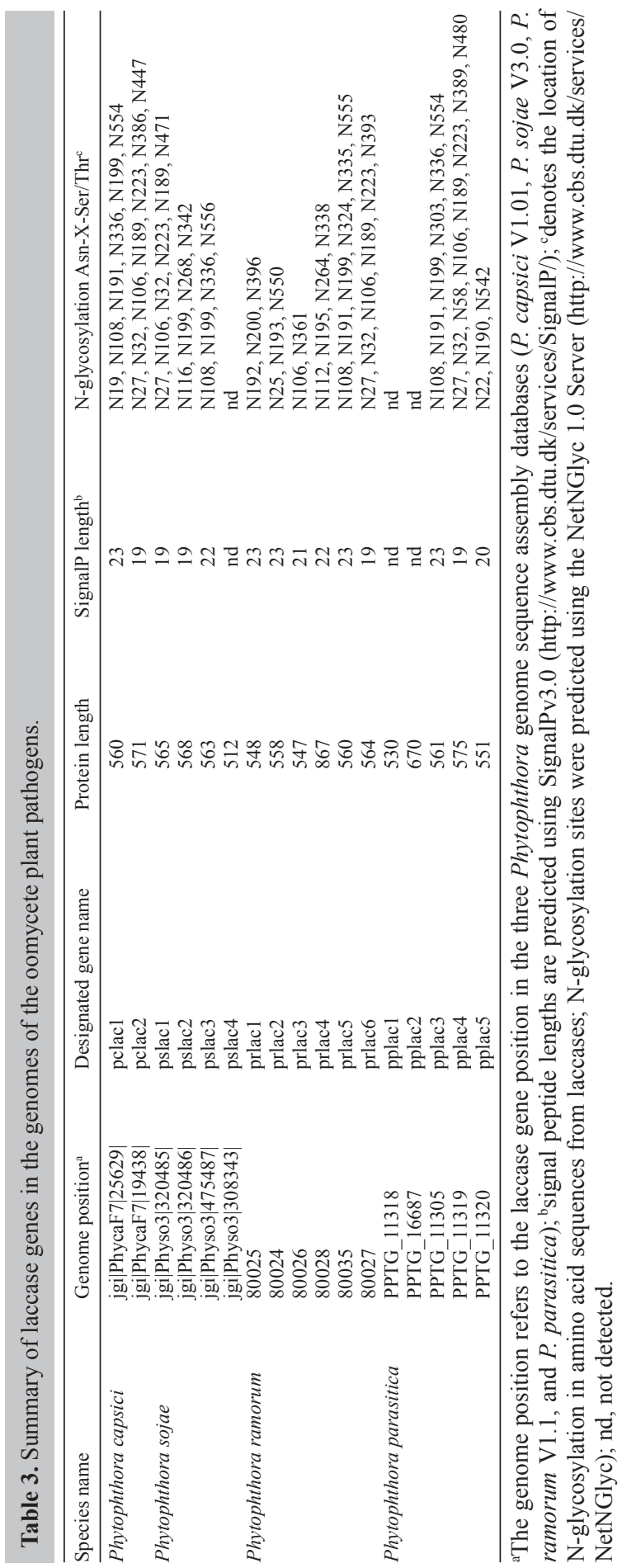


Oomycetes form a distinct phylogenetic lineage of fungus-like eukaryotic microorganisms that cause devastating diseases such as late blight of potato and sudden oak death. Many species of Phytophthora are plant pathogens of considerable economic importance. In this study, the four available genomes of the genera Phytophthora were analyzed. In $P$. capsici, a total of three genes were identified; in $P$. sojae, four were identified; and $P$. ramorum and $P$. parasitica presented six and five genes, respectively (Table 3 ).

\section{Sequence analysis}

Analysis of the coding sequences using the NCBI conserved domain database (CDD) server verified the $\mathrm{Cu}$-oxidase domain distribution among the 125 predicted proteins (data not shown). Only one candidate was selected from each genome to represent the $\mathrm{Cu}$-oxidase domain distributions. Line diagrams of these laccase sequences are shown in Figure 1.

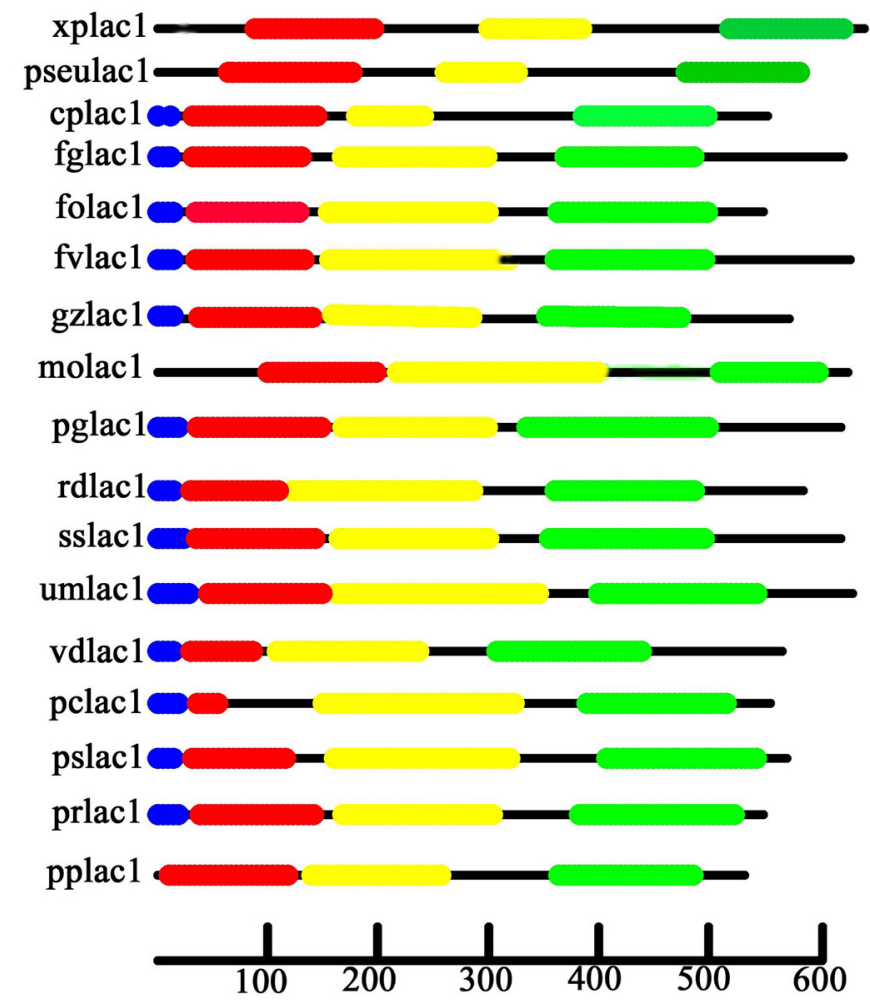

Figure 1. Predicted topology of laccase proteins. Protein domains are indicated as follows: blue, predicted signal peptide; red, $\mathrm{Cu}$-oxidase-3 domain; yellow, $\mathrm{Cu}$-oxidase domain; and green, $\mathrm{Cu}$-oxidase-2 domain. Line diagrams are drawn to scale. The origins of the laccases are, respectively: xplac1, Xanthomonas oryzae; pseulac1, Pseudomonas syringae; cplac1, Cryphonectria parasitica; fglac1, Fusarium graminearum $\mathrm{PH}-1$; folac1, Fusarium oxysporum f. sp lycopersici 4287; fvlac1, Fusarium verticillioides 7600-3; gzlac1, Gibberella zeae PH-1; molac1, Magnaporthe oryzae 70-15; pglac1, Puccinia graminis var. tritici; rdlac1, Rhizopus oryzae; sslac1, Sclerotinia sclerotiorum; umlac1, Ustilago maydis; vdlac1, Verticillium dahliae; pclac1, Phytophthora capsici V1.01; pslac1, Phytophthora sojae V3.0, prlac1, Phytophthora ramorum V1.1; and pplac1, Phytophthora parasitica. 
Laccase proteins from bacteria included approximately 457 to 638 amino acids (Table 1). Interestingly, none of these laccases had a signal peptide, which suggested that they were intracellular laccases. The putative laccases from fungi contained 488 to 1280 amino acids, and 114/121 laccase genes had 500 to 700 amino acids (Table 2). Only pglac 2 from $P$. graminis and umlac6 from U. maydis showed very long sequences with 1160 and 1289 amino acids, respectively. In particular, certain pathogenic fungi were considered to secrete extracellular laccases and also contain intracellular laccases according to our analyses using the SignalP v3.0 software. The vast majority of these putative laccases (80/114) contained a predicted signal peptide; their putative monobasic propeptide cleavage sites were located between amino acid residues 16 and 29. In addition, these 80 laccase genes showed numerous $\mathrm{N}$-glycosylation sites that varied among different species. Analysis of the amino acid sequences of the 17 putative laccases identified in oomycytes showed that their lengths were similar to the laccases of fungi, and most members (16/17) contained 500 to 700 amino acids. We also determined whether the protein sequences of the 17 Phytophthora laccases possessed secretory leader peptides using the SignalP v3.0 software. Following the parameter Markov-model (HMM) and signalP NN Mean Score, most of the members (14/17) contained a signal peptide and corresponding $\mathrm{N}$-glycosylation sites. For these 14 laccases, their putative monobasic propeptide cleavage sites were found to be present between amino acid residues 19 and 23, as shown in Table 3 .

Previously, four conservative regions have been characterized that are specific for all laccases (Kumar et al., 2003; Claus, 2004). One cysteine and ten histidine residues form a ligand environment of copper ions at the laccase active site and are present in these four conserved amino acid sequence domains. In order to examine whether these residues are conserved among the 125 laccases identified in this study, we conducted protein sequence alignments and compared amino acid sequences at the key sites, as shown in Figures 2 and 3. The data showed that most of the proteins had one cysteine and ten histidine residues at the conserved positions. These sequences present the strictly conserved residues: His79, His81, His123, His125, His420, His423, His425, His483, Cys484, His485, and His489 (numbered according to the cplac1 sequence). In addition, the following residues with more than $90 \%$ occurrence were found: Pro40, Gly41, Asn49, Pro54, Pro56, Gly62, Asp63, Asn70, Gly82, Asp91, Gly92, Gln98, Ile101, Pro103, Try109, Gly117, Trp120, Try121, Pro421, Gly426, Pro453, Arg457, Asp458, Asn475, Pro476, Gly477, Trp479, and Gly493. Most of these highly conserved and conservative residues are involved in the four copper-binding conserved domains of the typical laccase: regions I and II, as shown in Figure 2, and regions III and IV as shown in Figure 3; these regions correspond to regions L1-L4 as designated by Kumar et al. (2003). Of the putative proteins, 108/125 (86\%) possessed the four copper-binding conserved domains characteristic of typical laccases. Regions I (HxHG), II (HSH), III (HPxHxHG), and IV $(\mathrm{HCHxxH})$ are indicted in Figures 2 and 3. However, some amino acid residues differed from the consensus. For example, the second segment (HSH) was conserved in 81/108 sequences, whereas in the other sequences Ser was replaced by Ala, Gly, Pro, or Thr.

We also identified 17 sequences that contained incomplete typical laccase domains. In bacteria, ealac2 from E. amylovora only exhibited one conserved domain (region I) as shown in Figure 2, while pseulac1 and 2 from $P$. syringae did not possess region III, and the latter also lacked region IV. In fungi, a total of 13 genes contained two or three conserved domains, as shown in Figures 2 and 3; three members lacked regions III and IV (cplac16, pglac4, and vdlac4); while the rest lacked region I (folac13, folac14, fvlac13, gzlac3, pglac7, 
and vdlac1), region II (fglac11 and pglac4), or region IV (folac12 and molac5). In oomycetes, most putative genes presented intact conserved domains, and only one gene, pplac2 from $P$. parasitica, lacked region I.

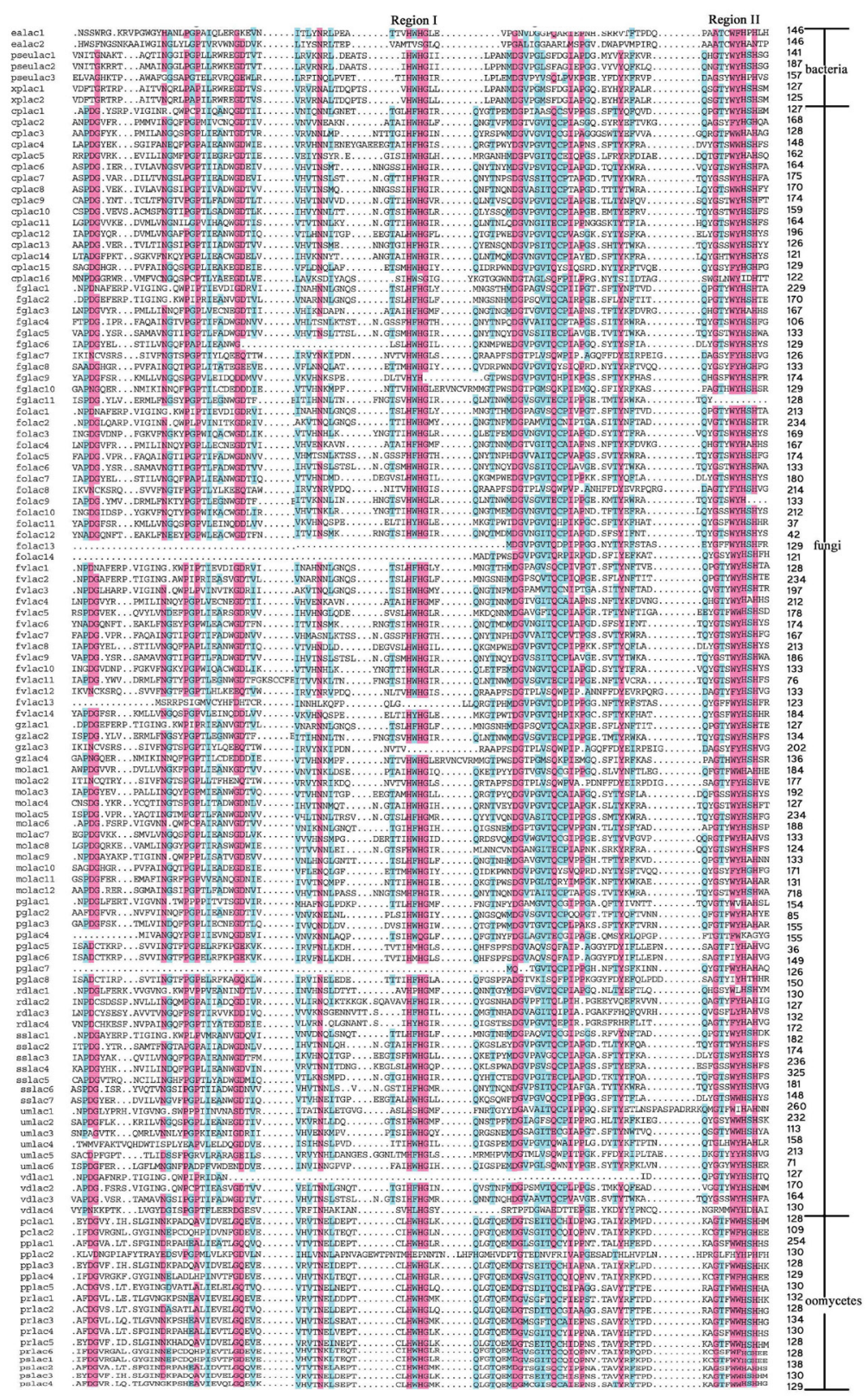

Figure 2. Protein sequence alignment of the 125 predicted laccase genes from plant pathogen genomes. The conserved copper-binding domains of typical laccase regions I and II are marked above the sequences, and conserved motifs and key functional histidine residues are indicated. All the sequences presented agree with those used in Tables 1-3. 


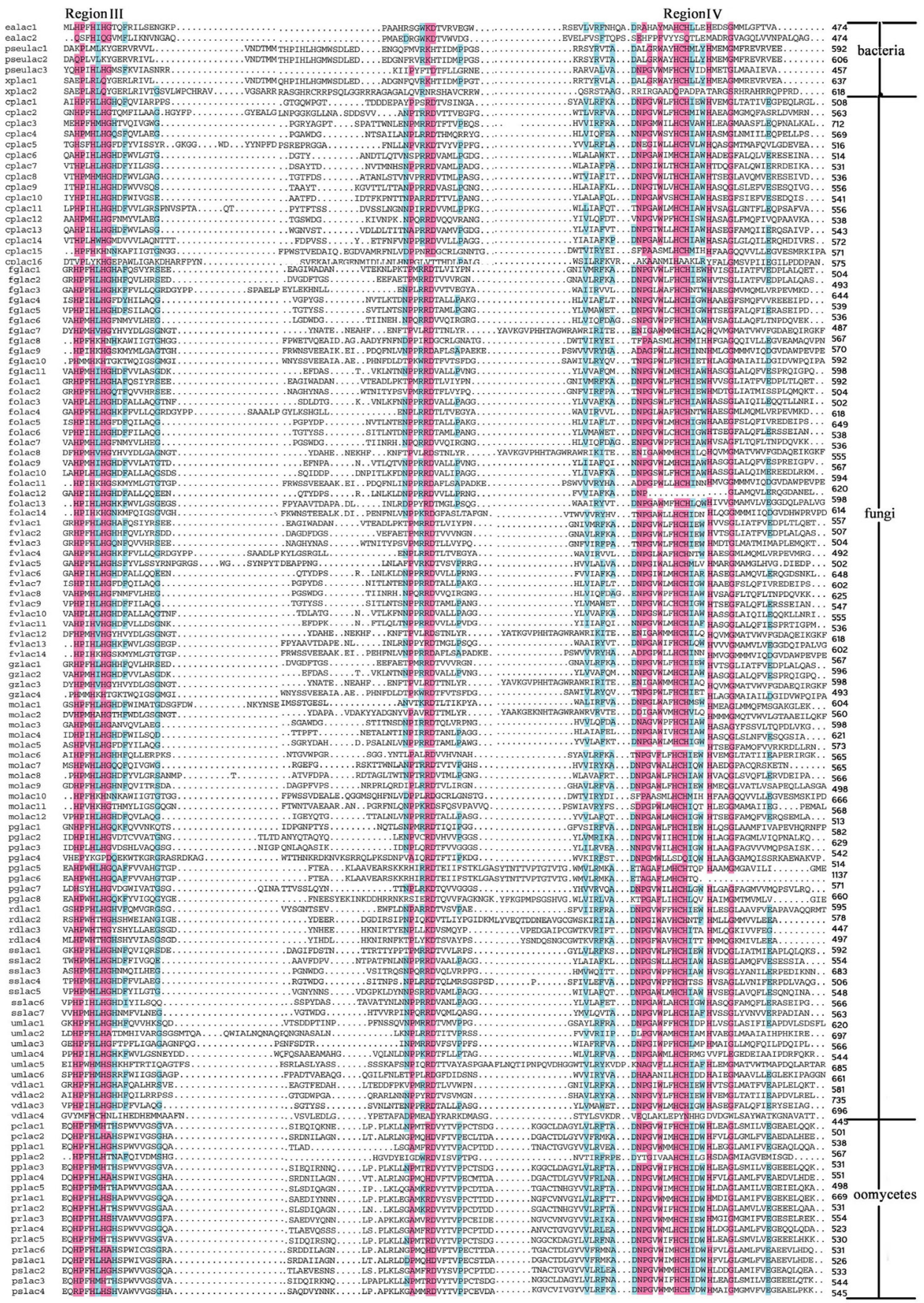

Figure 3. Protein sequence alignment of the 125 predicted laccase genes from plant pathogen genomes (continued). The conserved copper-binding domains of typical laccase: regions III and IV are marked above the sequences, and conserved motifs and key functional histidine residues are indicated. All the sequences presented agree with those used in Tables 1-3. 


\section{Phylogeny of laccase genes from plant pathogens}

To examine the relationships between the putative laccase genes from various sources, we constructed a phylogenetic tree on the basis of multiple-sequence alignment of the 125 putative laccase genes (as shown in Tables 1-3). The phylogenetic tree (Figure 4) shows the relationships among the selected 125 laccases. The laccases of bacteria, fungi, and oomycetes were separated into distinct groups, while the fungal genes separated into three main clades marked as fungi I, II, and III.

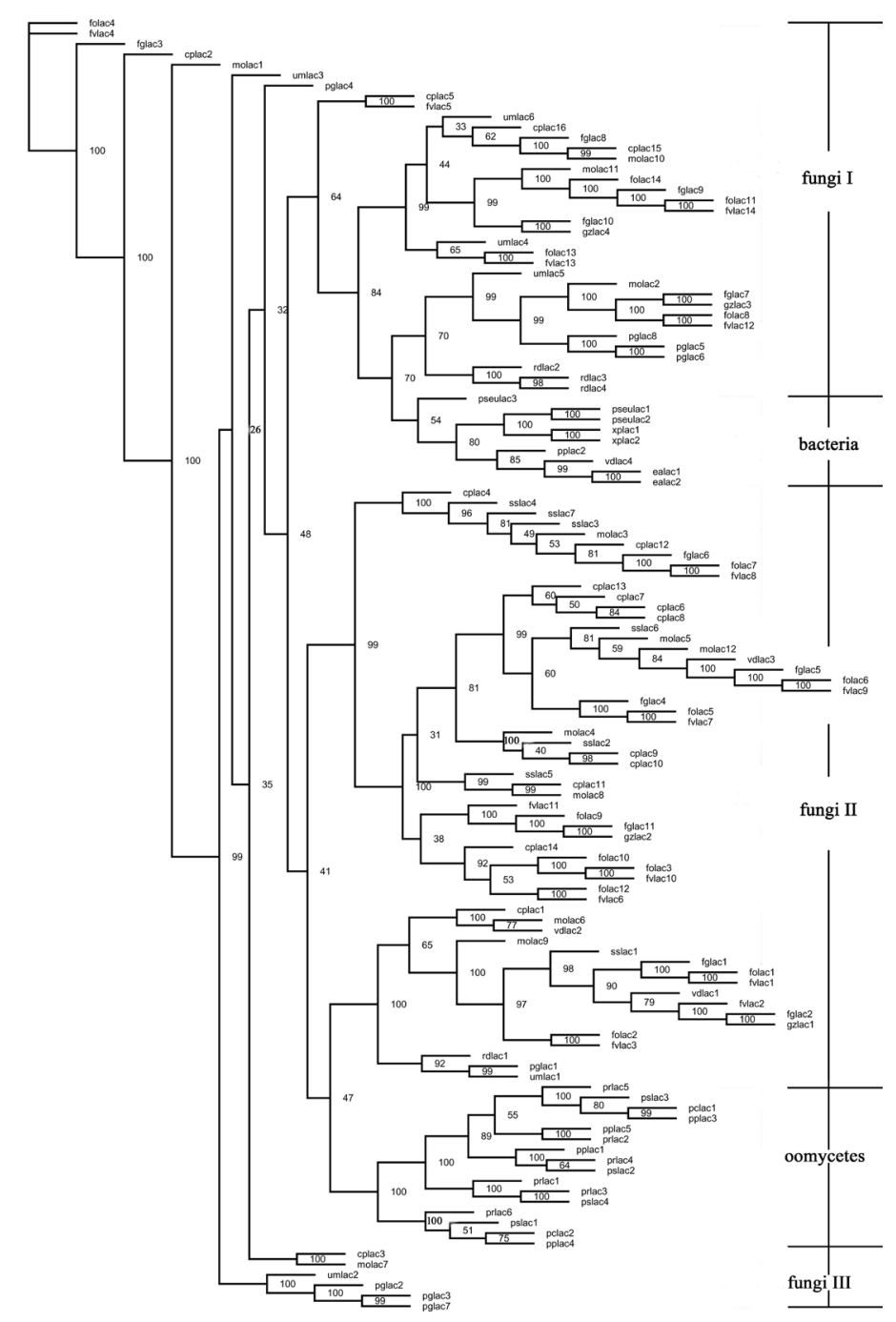

$$
100
$$

Figure 4. Phylogenetic tree constructed using PAUP 4.0 based on the protein sequences of each gene. The numbers at the nodes represent the percentage of their occurrence in 10,000 bootstrap replicates and the scale bar shows the number of amino acid differences per site. The amino acids of 125 predicted laccase genes originated from 4 bacterial species, 4 Phytophthora species, and 11 fungi species. All the sequences used agree with those in Tables $1-3$. 
The bacterial enzymes formed their own clade and also included the oomycete laccase pplac2 from P. parasitica and the fungal laccase vdlac4 from $V$. dahliae. The oomycete laccases were also well clustered into a clade. It should be pointed out that fungus clades I, II, and III were less compact since these were formed by more clusters that were not rooted in a single node (Figure 4).

\section{DISCUSSION}

In this study, we present as complete as is currently possible the structural and phylogenetic picture of plant pathogen laccases, based on analysis of putative amino acid sequences in the 20 publicly available plant pathogenic genomes by sequence alignment and construction of a phylogenetic tree. We identified 125 laccase genes in total, including seven in bacterial genomes, 17 in oomycete genomes, and 101 in fungal genomes. Most of the predicted protein models shared typical fungal laccase characteristics, possessing three conserved positions with one cysteine and ten histidine residues at these positions. Phylogenetic analysis illustrated that laccases from bacteria and oomycetes clustered efficiently into two different clades, while fungal laccases formed less compact clusters.

The distributions of the laccase genes of different origins varied considerably. In this study, fungi were found to contain a larger number of laccases than did oomycetes and bacteria. Among the species that were characterized as having a large number of laccase genes were C. parasitica, Fusarium, and M. oryzae, with 16, 39, and 13 genes, respectively. In other fungi, 9 genes each were identified in the filamentous ascomycetes Podospora anserina and Sordaria macrospora (Pöggeler, 2011), Aspergillus niger contained 6 (Ramos et al., 2011), and Chaetomium globosum had 4 (Hoegger et al., 2006). In oomycetes, candidate species of Phytophthora had five laccase genes on average. However, bacterial species possessed a low number of laccase genes, having only one to three genes in each genome. It should be pointed out that no laccase was retrieved in $P$. syringae dc3000, although three genes were found in $P$. syringae b728a, which is of the same genera. There is now increasing information regarding the diversity and distribution of laccases within bacteria including species living in extreme habitats (Ausec et al., 2011). Therefore, the roles of many laccases found in plant pathogens can be inferred in terms of protein structure as well as physiological function including substrate utilization, pigment formation, and stress resistance. It has been documented that some laccases from fungi are involved in morphogenesis and pathogenesis, as described earlier. Furthermore, the possibility that bacterial laccases play a role in biopolymer degradation has been suggested as well (Ahmad et al., 2010). Overall, however, very little is known directly about laccases from plant pathogens. It is therefore important to experimentally evaluate the functions of these putative proteins.

Sequence alignment of putative laccases showed that the copper-binding domains were highly conserved in most genes, even when the remaining amino acid sequences were of low similarity. The conserved copper-coordination sites had the sequences HxHG, HSH, HPxHxHG, and $\mathrm{HCxxH}$ localized near the $\mathrm{N}$ - and $\mathrm{C}$-termini, which was consistent with those of fungi (Fan et al., 2011). However, these conserved domains were not apparent in bacterial sequences. It was interesting to note that all of the laccase genes from oomycetes presented highly conserved domains in accordance with those of fungi. Thus, laccases from bacteria might have several prosperities that are not characteristic of the fungal or oomycete enzymes. 
In addition, the laccases from bacteria had no obvious signal peptides indicating that they were intracellular, which was consistent with current knowledge (Sharma et al., 2007). In contrast, most enzymes from fungi and oomycetes exhibited signal peptides, and were therefore considered to be extracellular enzymes. To our knowledge, the majority of known fungal laccases have extracellular activity, although intracellular laccases have also been identified (Baldrian, 2006). In fungi, the functions of extracellular laccases are related to the degradation of lignocellulose material, recycling of organic material, reduction of oxidative stress, and pathogenesis toward plants and animals, and have been extensively studied (Schouten et al., 2002; Baldrian, 2006). Of the 101 fungal laccases identified in this study, it was determined that 77 corresponded to extracellular laccases, whereas the other 34 were intracellular proteins (Table 2). Of the 17 genes identified in oomycetes, 82.4\% (14/17) related to extracellular activity (Table 3 ). On the other hand, according to the predictions generated in this study, glycosylation would usually be expected to occur in the extracellular enzymes, which suggested that these laccases were likely glycoproteins. The average glycosylation of laccases is usually between 10 and 25\% (Baldrian, 2006). Glycosylation influences enzyme secretion, and it has been suggested to play an important role in catalytic center stabilization, protection against hydrolysis, copper retention, and laccase thermal stability (Vite-Vallejo et al., 2009). Taken together, these results suggest that it would be important to experimentally evaluate the functions of the laccase gene families identified among these plant pathogens.

Phylogenetic analysis showed that the laccases from oomycetes and bacteria clustered into two distinct clades, which were well separated from the fungal laccases, while fungal laccases were included in three main clades. The phylogenetic relationships between these isoenzymes suggest structural similarities in terms of their regions and amino acids. It should be pointed out that all enzymes included in the tree were retrieved from genomes according to the same criteria, as shown in Tables 1-3. Because of this approach, the genes might represent different members of the MCO family. Therefore, it will be important to consider this aspect to generate a better definition of laccases in order to build more valid phylogenetic patterns that will provide a clearer idea of the evolutionary process of this enzyme and its functions among distinct species.

\section{Conflicts of interest}

The authors declare no conflict of interest.

\section{ACKNOWLEDGMENTS}

Research supported by the Project of Natural Science Foundation of Shanxi (\#2013021024-6) and the Doctoral Fund of Yuncheng University (\#YQ-2013014).

\section{REFERENCES}

Ahmad M, Taylor CR, Pink D, Burton K, et al. (2010). Development of novel assays for lignin degradation: comparative analysis of bacterial and fungal lignin degraders. Mol. Biosyst. 6: 815-821.

Ausec L, Zakrzewski M, Goesmann A, Schlüter A, et al. (2011). Bioinformatic analysis reveals high diversity of bacterial genes for laccase-like enzymes. PLoS One 6: e25724.

Baldrian P (2006). Fungal laccases - occurrence and properties. FEMS Microbiol. Rev. 30: 215-242.

Bendtsen JD, Nielsen H, von Heijne G and Brunak S (2004). Improved prediction of signal peptides: SignalP 3.0. J. Mol. 
Biol. 340: 783-795.

Cañero CD and Roncero MI (2008). Functional analyses of laccase genes from Fusarium oxysporum. Phytopathology 98 : 509-518.

Cázares-Garcia SV, Vázquez-Garcidueñas MS and Vázquez-Marrufo G (2013). Structural and phylogenetic analysis of laccases from Trichoderma: a bioinformatic approach. PLoS One 8: e55295.

Chung HJ, Kwon BR, Kim JM, Park SM, et al. (2008). A tannic acid-inducible and hypoviral-regulated laccase3 contributes to the virulence of the chestnut blight fungus Cryphonectria parasitica. Mol. Plant Microbe Interact. 21: 1582-1590.

Claus H (2004). Laccases: structure, reactions, distribution. Micron 35: 93-96.

Courty PE, Hoegger PJ, Kilaru S, Kohler A, et al. (2009). Phylogenetic analysis, genomic organization, and expression analysis of multi-copper oxidases in the ectomycorrhizal basidiomycete Laccaria bicolor. New Phytol. 182: 736-750.

Erwin DC and Ribeiro OK (1996). Phytophthora Diseases Worldwide. APS Press, St. Paul.

Fan F, Zhuo R, Sun S, Wan X, et al. (2011). Cloning and functional analysis of a new laccase gene from Trametes sp. 48424 which had the high yield of laccase and strong ability for decolorizing different dyes. Bioresour. Technol. 102: 3126-3137.

Felsenstein J (1985). Confidence limits on phylogenies: an approach using the bootstrap. Evolution 39: 783-791.

Feng BZ and Li PQ (2012). Genome-wide identification of laccase gene family in three Phytophthora species. Genetica 140: 477-484.

Feng BZ and Li PQ (2013). Cloning and expression of a novel laccase gene from Phytophthora capsici. J. Plant Pathol. 95: 417-421.

Feng BZ and Li P (2014). Cloning, characterization and expression of a novel laccase gene Pclac2 from Phytophthora capsici. Braz. J. Microbiol. 45: 351-357.

Hoegger PJ, Navarro-González M, Kilaru S, Hoffmann M, et al. (2004). The laccase gene family in Coprinopsis cinerea (Coprinus cinereus). Curr. Genet. 45: 9-18.

Hoegger PJ, Kilaru S, James TY, Thacker JR, et al. (2006). Phylogenetic comparison and classification of laccase and related multicopper oxidase protein sequences. FEBS J. 273: 2308-2326.

Kilaru S, Hoegger PJ and Kües U (2006). The laccase multi-gene family in Coprinopsis cinerea has seventeen different members that divide into two distinct subfamilies. Curr. Genet. 50: 45-60.

Kumar SV, Phale PS, Durani S and Wangikar PP (2003). Combined sequence and structure analysis of the fungal laccase family. Biotechnol. Bioeng. 83: 386-394.

Kwon SI and Anderson A (2001). Laccase isozymes: Production by an opportunistic pathogen, a Fusarium proliferatum isolate from wheat. Physiol. Mol. Plant Pathol. 59: 235-242.

Larkin MA, Blackshields G, Brown NP, Chenna R, et al. (2007). Clustal W and Clustal X version 2.0. Bioinformatics 23 : 2947-2948.

Lettera V, Piscitelli A, Leo G, Birolo L, et al. (2010). Identification of a new member of Pleurotus ostreatus laccase family from mature fruiting body. Fungal. Biol. 114: 724-730.

Levasseur A, Saloheimo M, Navarro D, Andberg M, et al. (2010). Exploring laccase-like multicopper oxidase genes from the ascomycete Trichoderma reesei: a functional, phylogenetic and evolutionary study. BMC Biochem. 11: 32.

Litvintseva AP and Henson JM (2002). Cloning, characterization, and transcription of three laccase genes from Gaeumannomyces graminis var. tritici, the take-all fungus. Appl. Environ. Microbiol. 68: 1305-1311.

Marchler-Bauer A and Bryant SH (2004). CD-Search: protein domain annotations on the fly. Nucleic Acids Res. 32: W327-W331.

Marchler-Bauer A, Anderson JB, Chitsaz F, Derbyshire MK, et al. (2009). CDD: specific functional annotation with the Conserved Domain Database. Nucleic Acids Res. 37: D205-210.

McGuirl MA and Dooley DM (1999). Copper-containing oxidases. Curr. Opin. Chem. Biol. 3: 138-144.

Pöggeler S (2011). Evolution of multicopper oxidase genes in coprophilous and non-coprophilous members of the order Sordariales. Curr. Genomics 12: 95-103.

Quintanar L, Stoj C, Taylor AB, Hart PJ, et al. (2007). Shall we dance? How a multicopper oxidase chooses its electron transfer partner. Acc. Chem. Res. 40: 445-452.

Ramos JA, Barends S, Verhaert RM and de Graaff LH (2011). The Aspergillus niger multicopper oxidase family: analysis and overexpression of laccase-like encoding genes. Microb. Cell Fact. 10: 78.

Rigling D and Van Alfen NK (1991). Regulation of laccase biosynthesis in the plant-pathogenic fungus Cryphonectria parasitica by double-stranded RNA. J. Bacteriol. 173: 8000-8003.

Rigling D and Van Alfen NK (1993). Extra- and intracellular laccases of the chestnut blight fungus, Cryphonectria parasitica. Appl. Environ. Microbiol. 59: 3634-3639.

Rodriguez Couto S and Toca Herrera JL (2006). Industrial and biotechnological applications of laccases: A review. Biotechnol. Adv. 24: 500-513. 
Schouten A, Wagemakers L, Stefanato FL, van der Kaaij RM, et al. (2002). Resveratrol acts as a natural profungicide and induces self-intoxication by a specific laccase. Mol. Microbiol. 43: 883-894.

Sharma P, Goel R and Capalash N (2007). Bacterial laccases. World J. Microbiol. Biotechnol. 23: 823-832.

Vite-Vallejo O, Palomares LA, Dantán-González E, Ayala-Castro HG, et al. (2009). The role of N-glycosylation on the enzymatic activity of a Pycnoporus sanguineus laccase. Enzyme Microb. Technol. 45: 233-239.

Wahleithner JA, Xu F, Brown KM, Brown SH, et al. (1996). The identification and characterization of four laccases from the plant pathogenic fungus Rhizoctonia solani. Curr. Genet. 29: 395-403.

Yoon HS, Hackett JD, Pinto G and Bhattacharya D (2002). The single, ancient origin of chromist plastids. Proc. Natl. Acad. Sci. U. S. A. 99: 15507-15512.

Zhao J and Kwan HS (1999). Characterization, molecular cloning, and differential expression analysis of laccase genes from the edible mushroom Lentinula edodes. Appl. Environ. Microbiol. 65: 4908-4913.

Zhu X and Williamson PR (2004). Role of laccase in the biology and virulence of Cryptococcus neoformans. FEMS Yeast Res. 5: 1-10.

Zhu X, Gibbons J, Garcia-Rivera J, Casadevall A, et al. (2001). Laccase of Cryptococcus neoformans is a cell wallassociated virulence factor. Infect Immun. 69: 5589-5596. 